\title{
Subgrains, micro-twins and dislocations characterization in monolike Si using TEM and in-situ TEM
}

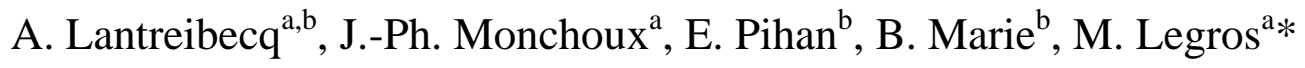 \\ ${ }^{a}$ CEMES-CNRS-Université de Toulouse, 29 rue J. Marvig, , 31055 Toulouse, France . \\ ${ }^{\mathrm{b}}$ Univ. Grenoble Alpes, INES, F-73375 Le Bourget du Lac, France \\ CEA, LITEN, Department of Solar Technologies, F-73375 Le Bourget du Lac, France
}

\begin{abstract}
A critical aspect in the development of mono-like Silicon for photovoltaic (PV) application lies in the control of its defect microstructure. While micro-twins do not seem to represent a major concern regarding electrical activity, some dislocations and low angle grain boundaries need to be avoided in the growing process because of their recombination properties. Here, we have studied how these defects grow and interact together in order to understand what are the mechanisms responsible for their presence in the cast ingots. We show that, in our specific convex liquid-solid interface growth mode, micro-twins probably originate from this interface while dislocations and subgrains build up both by epitaxy at the solid liquid interface and through dislocation-boundary interactions in the solidified region. Post-growth TEM characterization allowed identifying constitutive dislocations of some subgrain boundaries for the case of [001] seeds. In-situ TEM straining of Si at high temperature also allowed measuring dislocation mobility at several temperatures. Those are compared to the dislocation velocities in pure Czochralski Si found in literature.
\end{abstract}

(C) 2017 Elsevier Ltd. All rights reserved.

Selection and/or Peer-review under responsibility of Extended Defects In Semiconductors 2016 (EDS 2016).

Keywords: PV Si; dislocations; sub-grain boundaries; micro-twin; growth; defects; Transmission electron microscopy (TEM); in-situ TEM

\section{Introduction}

Monolike silicon (ML-Si) is a potentially ideal candidate material for high efficiency solar cells, when no low angle grain boundaries are present [1]. It combines a low cost production of wafers with a photovoltaic efficiency that is close to that of Czochralski $(\mathrm{Cz})$ Silicon [2]. Large size ingots can be produced by directional solidification casting on dislocation-free $\mathrm{Cz}$ seeds. The process can theoretically lead to dislocation-free ingots [3].

Anyway, many macrodefects having an impact on solar cell efficiency have been found in such ingots. Parasitic mc$\mathrm{Si}$ (or large twinned) grains and large subgrains domains originating from inappropriate seed junctions may for instance appear and multiply essentially on the edges of the ingots and degrade locally the PV efficiency of Si (see for example [4], [5]). When [001] growth initiates far from any inappropriate seed junction or multicrystalline silicon (mc-Si) sources [2], the main residual defects are usually dislocations. As in [6], we distinguish dislocation clusters, that we call subgrain boundaries (SGBs) and can reach densities of over $10^{6} \mathrm{~cm}^{-2}$ and background dislocation densities in the range of $10^{4} \mathrm{~cm}^{-2}$. Subgrains occurrence in such case could arise from seed deformations

\footnotetext{
* Corresponding author. Tel.: +33-(0)5-62-25-7842; fax: +33-(0)5-62-25-7999.

E-mail address: marc.legros@cemes.fr
} 
or indentations [7-9] and/or over bulk thermomechanical stress [10] as in Bridgman growth of II-VI and III-V semiconductors [11].

Thermomechanical stresses can arise from thermal gradients or edge effects. The multiplication of dislocations is usually described by the Alexander-Haasen (AH) model [12] or the AH model extended to multiple slip system called Haasen-Alexander-Sumino (AHS) model [13], [14]. In both of them, multiplication depends on the velocities of dislocations. These dislocation velocities were established from various $\mathrm{Cz}$ and Float zone (FZ) silicon ingots [15-18]. Because the cooling of large size ingots takes a long time, solidified silicon remains in a temperature range where dislocations are mobile for several hours. It is therefore important to know their velocities in monolike ingots to know if AH and AHS models can still apply. The determination of the dislocation mean free path is also a good indication to compare it to cells and subgrains sizes that form in the interior of the ingot. If the mean free path is much larger than these structures, it means that, during the cooling of the ingot, dislocation glide will be hindered by other dislocations, subgrains and micro-twins, contributing to their stabilization by local reactions. Dislocations pileups resulting from these obstacles may also lead to stress concentration. In the upper temperature regime, such stress concentrators may activate dislocation sources or dislocation transmission across obstacles such as twins or subgrain boundaries. In any case, they will not be able to fully relax the internal stresses during lower temperature cooling. Stress concentrators structures have been found in multicrystalline silicon growth that experience similar thermal history as ML-Si ingots. In [19], it was shown that stacking faults act as barriers to dislocation glide. The observed microstructure in dislocations rich regions in mc-Si appears to be formed through a recovery process (rather than plastic slip)[20]. Lomer dislocations have also been identified at small-angle grain boundaries in mc-Si [21]. From reference [22], it can also be deduced that dislocations substructures might strongly depend on each grain orientation. The electrical activity correlates with the amount of misorientation of a given SGB, which reflects the fact that larger angles correspond to denser dislocations. Even if this remains to be validated at a larger scale in production mono-like ingots, EBSD results specifically targeting at the rotations around [001] growth axis electrically active subgrain domains are characterized by a mosaic rotation of the crystal structure around the growth axis for recent industrial-scale experiments [8].

We have used both EBSD measurements in zones that were identified as electrically active (where misorientations were detectable) and TEM in SGBs identified at smaller scale, where misorientations and dislocation densities are smaller. We will show that misorientations in SGBs, even with different amplitudes can be explained with the same dislocations structures. Overall, this work aims at identifying dislocations types in SGB in the [001] oriented growth case, to improve our knowledge on dislocation mobility in directionally solidified silicon and generally to identify stress concentrators structures such as $2 \mathrm{D}$ defects.

\section{Experimental procedure}

\subsection{Ingot}

A laboratory scale G2 85kg directionally solidified silicon ingot (2013 INES Standards) grown on Cz seeds was used for this study. The four [100] orientated seeds were arranged as a variant of patent FR13 54761. With this weight, the ingot height is close to industrial-size ingots height which allows possible development of subgrain during growth and cooling. The ingot is boron-doped with resistivities in the range $0.8-1.4 \mathrm{Ohm} . \mathrm{cm}$. In this type of growth, an almost constant vertical thermal gradient of $11-13 \mathrm{~K} / \mathrm{cm}$ is generated in the solid silicon for typical growth rates of $10-15 \mathrm{~mm} / \mathrm{h}$. The solid/liquid interface shape is convex meaning that a given time during melting and solidification, the solid liquid interface position is higher in the center than at the 4 corners. The growth interface deflection between center and corners increases during growth from bottom $(\approx 6-8 \mathrm{~mm})$ to the top $(\approx 30$ $40 \mathrm{~mm}$ ) allowing seed orientation preservation toward the ingot height. At brick scale, PL characterization reveals that electrically active SGBs affect mainly the quality of top sides and corners of the ingot. The sample impurity content in interstitial oxygen $\left[\mathrm{O}_{\mathrm{i}}\right]$ and substitutional carbon $\left[\mathrm{C}_{\mathrm{s}}\right]$ is measured by FTIR (Fourier Transform InfraRed spectroscopy): $\left[\mathrm{C}_{\mathrm{s}}\right]$ is in the range of $3-8 \times 10^{17} \mathrm{~cm}^{-3},\left[\mathrm{O}_{\mathrm{i}}\right]$ in the range of $3-14 \times 10^{16} \mathrm{~cm}^{-3}$ and the nitrogen content is unknown but considered as incorporated from saturated or oversaturated melt at the solid/liquid interface. The main 
challenge in this study is to go down across the different scales, to prepare samples for the various characterization techniques along the path and to retain the meaningful microstructural features. To do so, we have adopted the following procedure, sketched in Fig. 1. G2 ingots are cut in 4 bricks among which one is sawn in 367 wafers or in 26 square slices $156^{2} \times 7 \mathrm{~mm}^{3}$ once the top and bottom regions have been discarded. At this stage, the slices are inspected by uncalibrated room temperature photoluminescence (PL) then some slices are polished, optically inspected and etched to reveal dislocations and subgrains. 25 smaller zones are then cut in each slice and eventually polished again. In the following, $\mathrm{h}$ will indicate, in $\mathrm{cm}$, the height at which the sample has been taken in the ingot, starting from the mold/Si interface.

\subsection{Sample EBSD preparation and EBSD on a large area}

Long range misorientations across the subgrains and SGBs were measured through Electron BackScatter Diffraction (EBSD) measurements on cm-scale samples ("zones" in Fig.1). The sample surface was subjected to a careful chemical-mechanical polishing. They were observed in a Nova NanoSEM 630 FEG-SEM from FEI equipped with a Nordlys Nano detector from Oxford Instruments. This tool allows the possibility to map zones up to $20 \mathrm{x} 70 \mathrm{~mm}^{2}$ with an approximate accuracy on the order of 0.1 degrees.

\subsection{TEM sample preparation}

In the small zones previously defined, TEM samples are either picked-up using FIB (Focused Ion Beam) lift-out and polishing or further sliced and polished to prepare $1 \cdot x 3 \mathrm{~mm}$ rectangular samples for in-situ TEM straining.

Three types of sample preparation methods were used in this study: mechanical abrasion on SiC papers followed by dimpling and ion milling at $5 \mathrm{keV}$ in a PIPS ${ }^{\circledR}$ polisher, tripod polishing or FIB lamella extraction using a Helios 600i from FEI.

High temperature straining experiments were carried out on polished Si rectangles extracted from slice 7, zone 43. At this height of $\mathrm{h}=89 \mathrm{~cm}$, the impurity content is $\left[\mathrm{C}_{\mathrm{s}}\right]=3.5 \times 10^{17} \mathrm{~cm}^{-3}$ and $\left[\mathrm{O}_{\mathrm{i}}\right]=7 \times 10^{16} \mathrm{~cm}^{-3}$. They were prepared as follows. $3 \times 1 \times 0.5 \mathrm{~mm}^{3} \mathrm{Si}$ rectangles were first cut using diamond wire saw and then polished down to a thickness of about 30 to $50 \mu \mathrm{m}$. They were then dimpled using $3 \mu \mathrm{m}$ and $1 \mu \mathrm{m}$ diamond paste until optical transparency. Before being ion milled in a Gatan PIPS at $5 \mathrm{kev}$ and $3 \mathrm{keV}$, they were glued on custom-made Mo or Ta grids using alumina-based high temperature cement (Cotronics 908 ®). 

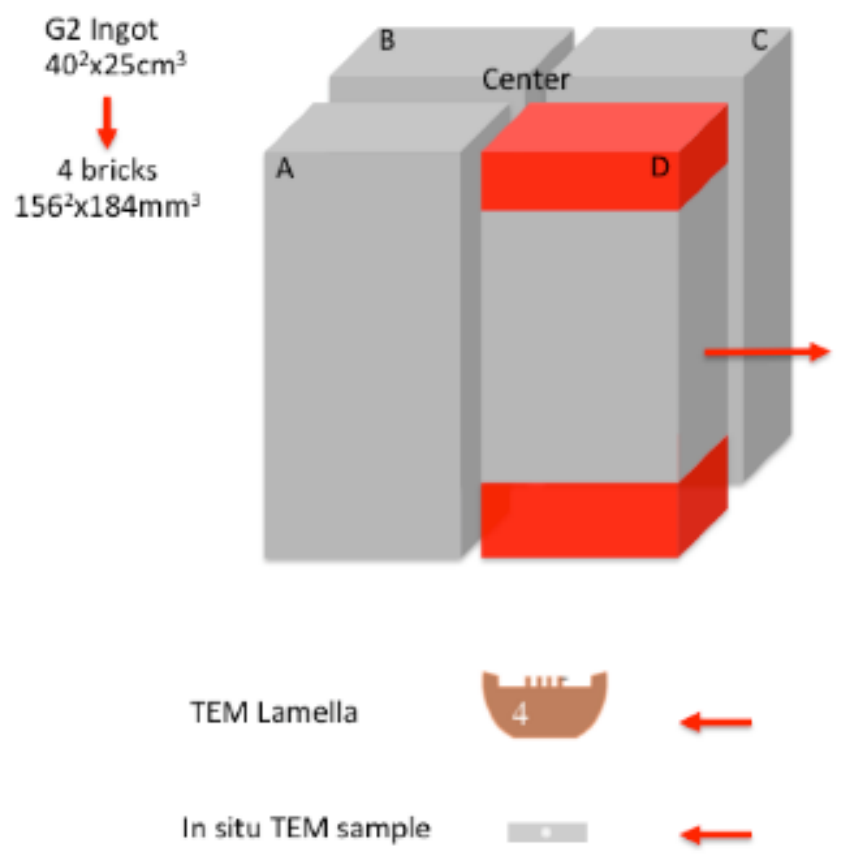

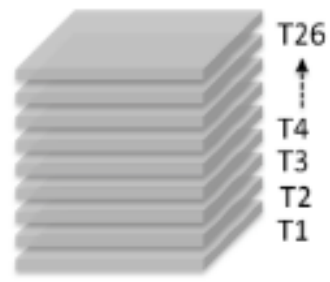

\section{Zones / slice}

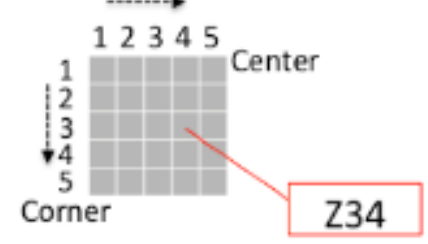

Fig. 1. Slicing of a G2 ingot ( $40 \times 40 \times 24.5 \mathrm{~cm} 3)$ into 4 bricks (labelled A-C, dimensions $156 \times 156 \times 184 \mathrm{~cm} 3)$ and down to a TEM lamella (FIB or tensile sample). After discarding the top and bottom $25 \mathrm{~mm}$ of each brick (red parts), the remaining core is sliced in $26.7 \mathrm{~mm}$ thick slices (sometimes in $300 \mu \mathrm{m}$ thick wafers). Each slice is then further separated in 25 smaller zones (31 x $31 \mathrm{~mm}$ squares) numbered from Z11 to Z55. TEM samples are selected in these zones. Those are either TEM lamella, typically $20 \times 5 \times 0.2 \mu \mathrm{m}^{3}$, extracted by FIB and welded to an omniprobe grid, or in-situ TEM samples, $1 \times 3 \times 0.3 \mathrm{~mm}^{3}$ that are further dimpled and ion milled to electron transparency. These in-situ rectangular samples are glued with a high temperature alumina-based cement on Ta or Mo tensile grids.

\subsection{TEM and in-situ TEM high temperature straining}

TEM observations were carried out in bright and dark field imaging, following two beam conditions [23] and using simple tilt and tilt-rotation TEM holders. Orientation of the sample inside the TEM relied on two-beam condition diffractions (see Fig. 4 and Annex 1).

The initial plastic deformation of TEM samples was performed at high temperature $\left(\sim 1050^{\circ} \mathrm{C}\right)$ to produce enough dislocations in the foil to overcome the first yield peak of Si [18], [24], [25] before being cooled down to the temperature of test. Here, 4 temperatures were tested: $980,850,720$ and $690^{\circ} \mathrm{C}$. Dislocation velocity measurements were made in relaxation mode. Strain increments were applied to the sample and observations were made essentially during the stress relaxation following these steps. The high temperature TEM holder used for this study is a custom made equipment where temperatures are pre-calibrated in function of the electrical power injected in the resistances bordering the sample (Fig. 2).

Images were taken in a JEOL 2010 microscope operating at 200kV. Videos were captured on a DVD/HD recorder through a Megaview II (SIS-Olympus) CCD camera operating at $22 \mathrm{im} / \mathrm{s}$.

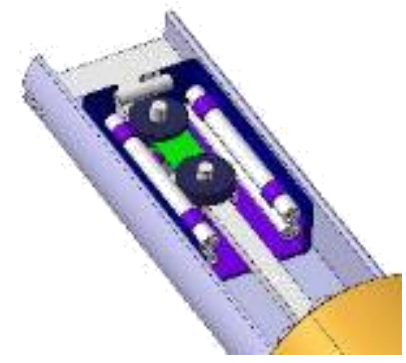


Fig. 2. Head of the high temperature holder used for in-situ TEM Si straining. 5 resistances (white cylinders) border the sample (green) that is hold between two Mo washers (dark grey) in this configuration. Ta foils (purple) on bottom and top (not represented here) serve to concentrate the heat on the sample. An alumina mobile jaw (white rectangle) serves to apply the load.

\section{Results}

\subsection{Subgrain boundaries}

Fig. 3 represents the surface of a sample selected at mid-ingot height $\mathrm{h}=110 \mathrm{~cm}$, after mechanical polishing and

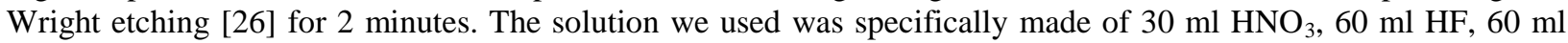
acetic acid, $1 \mathrm{~g} \mathrm{CrO}_{3}, 2 \mathrm{~g}$ of hydrated copper nitrate $\left(\mathrm{Cu}\left(\mathrm{NO}_{3}\right)_{2}-3 \mathrm{H}_{2} \mathrm{O}\right)$ and $60 \mathrm{ml}$ of DI water.

A typical circular subgrain boundary (SGB) with a radius close to $150 \mu \mathrm{m}$ and a total length of $1100 \mu \mathrm{m}$ is revealed. Subsequent images Fig. 3b and c are close-up images of the circular SGB taken in the blue and green squares of Fig. 3a. It can be seen that these portions of GB have a very similar and representative structure made of two types of etch pits along the grain boundary. High resolution SEM Fig. $3 \mathrm{~d}$ reveals that larger etch pits (size $\approx 250 \mathrm{~nm}$ ) are separated by very dense and small etch pits $($ size $\approx 100 \mathrm{~nm})$ that are very regularly spaced and distant $90 \mathrm{~nm}$ from each other. We assume here that each etch pit type corresponds to a single dislocation family that we label d1 (small etch pits) and $\mathrm{d} 2$ (larger etch-pits). This hypothesis will be confronted to TEM observations of the SGB in a later section. Etch pits $\mathrm{d} 2$ are irregularly spaced, with a separation distance varying between 0.5 and $1 \mu \mathrm{m}$. The probability that both etch pits types correspond to other microstructural features such as precipitates is unlikely given their very distinct size and the fact that they all align perfectly with the grain boundary.

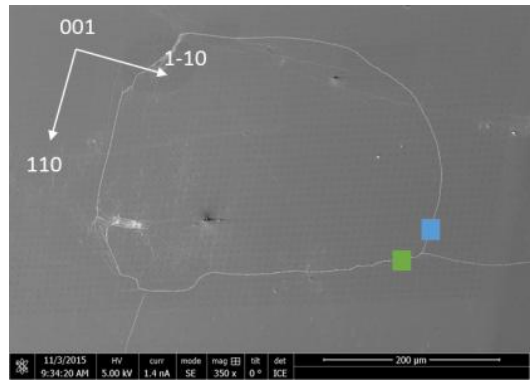

a)

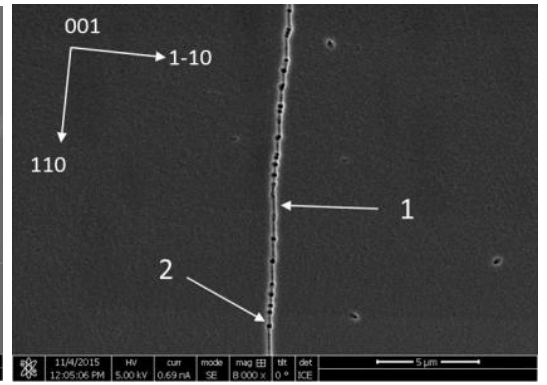

b)

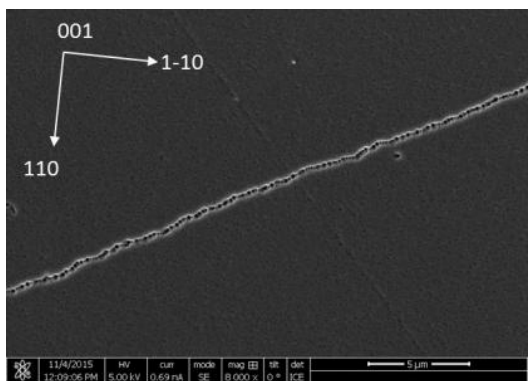

c)

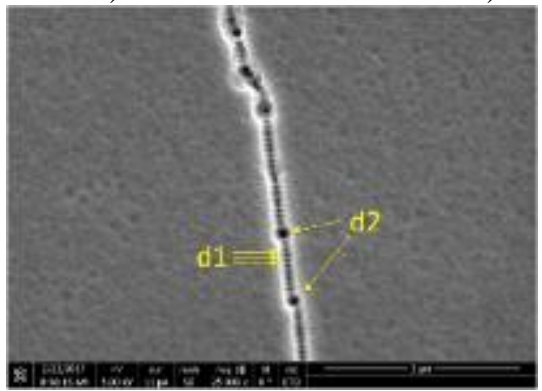

d)

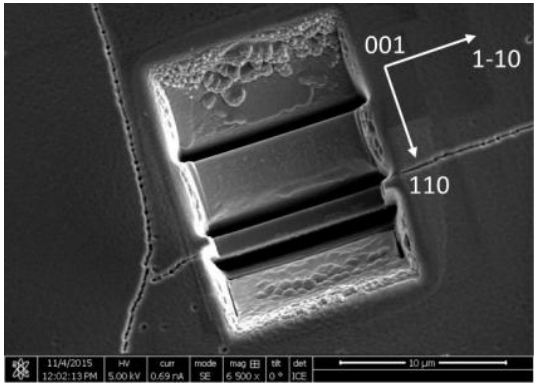

e)

Fig. 3. SEM images of a subgrain observed at mid height $(\mathrm{h}=110 \mathrm{~cm}$ in the zone D_T10_Z43) after being revealed by Wright etching. a) General view of the subgrain. b) and c) are magnified images of the blue and green squares in a), respectively, showing the etch pits due to the SGB dislocations preferential etching. d) High resolution image of the etch pits showing two distinct populations: d1 (smaller, denser) and d2 (larger, less dense). e) Same zone after the extraction of the TEM FIB lamella observed in Fig. 4. 


\subsection{Microstructural characterization of sub-grain boundaries}

Fig. 4 is a set of dark field TEM micrographs taken in the FIB foil that was extracted from the SGB location shown in Fig. 3e. The different diffraction vectors used can be located in the corresponding stereographic projection, Fig. 4b. Three different families of a/2[110] dislocations are revealed, labelled $\mathrm{d} 1, \mathrm{~d} 2$ and $\mathrm{d} 3$, and whose Burgers vectors have been analysed using the $\overrightarrow{\mathrm{g}} \cdot \overrightarrow{\mathrm{b}}=\overrightarrow{0}$ invisibility criterion.

The densest family of dislocations, labelled d1 (Fig. 4d), is aligned along the [001] growth axis and is the main constituent of the SGB. As can be seen in Fig. 4a and c, this set of dislocations is out of contrast using $\overrightarrow{\mathrm{g}}=$ [004] and $\overrightarrow{\mathrm{g}}=$ [113] diffraction vectors (Fig. 4a and c, respectively). They are therefore of a/2[1-10] type, which indicates that they are perfect edge dislocations. These dislocations are very evenly separated, about $90 \mathrm{~nm}$ apart. Two other sets of dislocations (d2 and d3) are visible in this SGB. Complementary dislocation analysis for these second and third family is given in the Annex 1 of the supplementary material. This configuration with at least 3 families of dislocations, the alignment of the densest one along [001] and the segments aligned along possible $\{111\}$ glide planes has been found in the three foils extracted and are there typical of SGBs at this height of the ingot. When comparing these findings with those obtained from etch pit observations (Fig. 3), it is clear that $\mathrm{d} 1$ correspond to the densest family of dislocation (smaller etch pits) and that the $\mathrm{d} 2$ family correspond very probably to the larger etch pits. At this point, it is not completely clear if the $\mathrm{d} 3$ family (the one with the lowest density) would give an etch pit signature different from the $\mathrm{d} 2$ family.

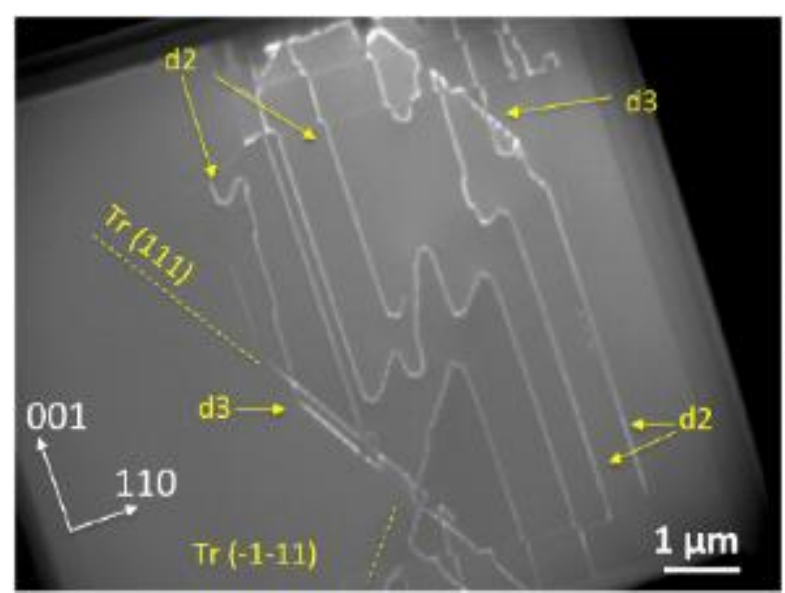

a)

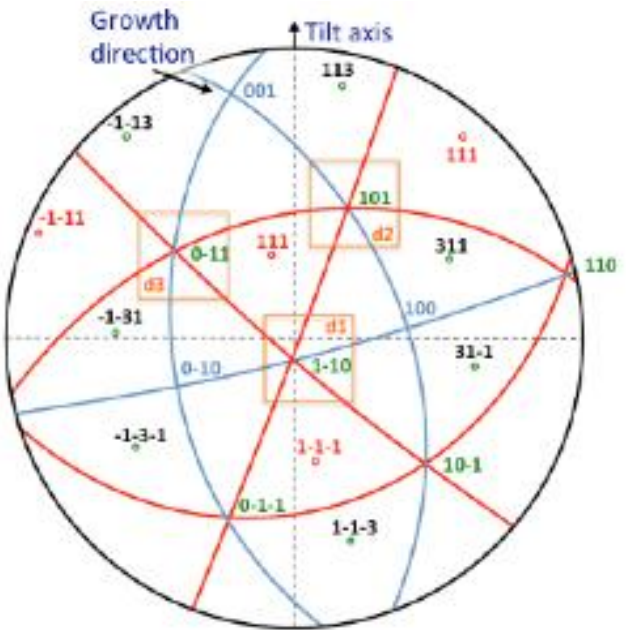

b) 


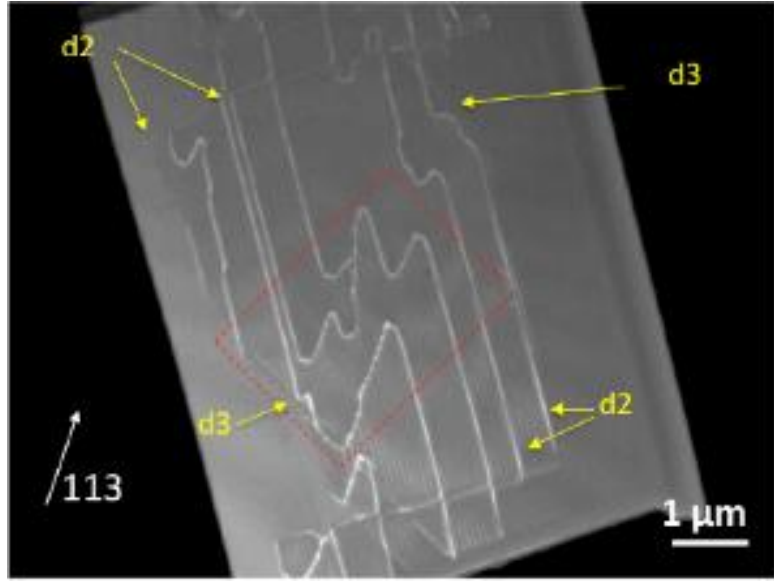

c)

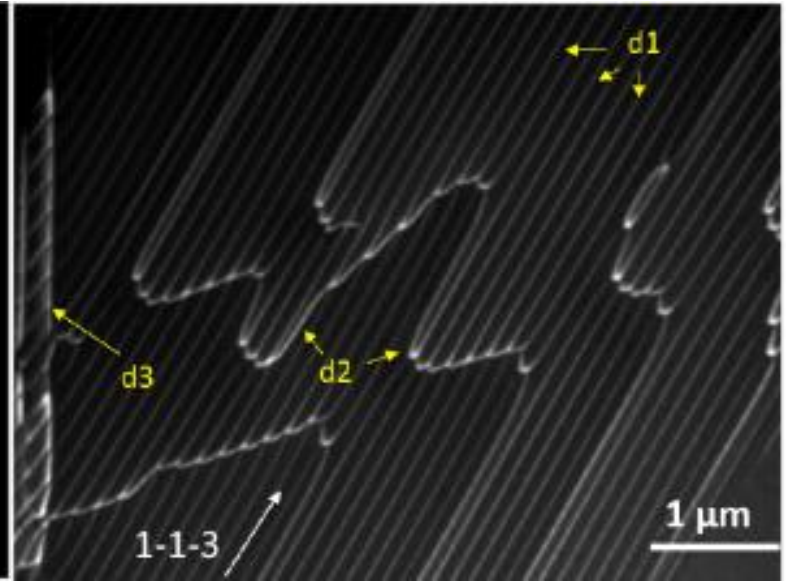

d)

Fig. 4. Set of TEM micrographs taken in the FIB foil extracted from the location in Fig. $3 \mathrm{~d}(\mathrm{~h}=110 \mathrm{~cm})$ and showing the 3 dislocation families present in a SGB. These families are labelled d1, d2, d3. a) $\vec{g}=[004]$, dislocation family $\mathrm{d} 1$ is out of contrast, b) corresponding stereographic projection of the foil in a) before tilting to $\left.+25^{\circ}(\overrightarrow{\mathrm{g}}=[004]), \mathrm{c}\right) \overrightarrow{\mathrm{g}}=[113]$, d) is a close up of the zone in the red dashed rectangle in c) and is captured with $\mathrm{g}=1-1-3$ (the foil has been rotated $\approx 50^{\circ}$ in plane to capture this image). In the stereographic projection, blue planes correspond to (001) type planes, red planes to (111). The Burgers vectors $(<110\rangle$ type, written in green) surrounded by a square are those identified as $\mathrm{d} 1$, d2 and $\mathrm{d} 3$ families. Other vectors such as [113]-types (used for imaging conditions) are labelled in black.

In parallel, EBSD measurements have been performed at a larger scale (here $1.2 \mathrm{~cm}$ wide maps) along the growth direction on these SGB from vertical cuts. Fig. 5 is an example of such maps taken in this ingot at the top corner of another brick where electrically active SGBs can be observed by PL measurements. Xo corresponds here to the [001] axis and deviates only slightly from the normal to the solid/liquid interface (the local interface deflection during growth is probably associated to SGBs propagation direction). No significant misorientation can be measured by EBSD along the Xo axis. This means that the observed misorientations on the Zo orientation maps of Fig. 5 (similar ones are obtained in the Yo direction) characterize mosaic type rotations around the [001] axis with variable absolute values and signs (black and purple arrows domains have opposite sign rotations). Most of the misorientation is seen across the SGBs (line 1-4) and spans over a total of about $3.5^{\circ}$. Each domain shows however a smaller misorientation with its closest neighbour, typically less than $1^{\circ}$ to $2.1^{\circ}$. Along these domains, $0.6^{\circ}$ to $1.5^{\circ}$ are found (black and purple arrows in Fig. 5b). The transition between each domain is sharp as stated by the steps in Fig. 5d), which indicates the presence of condensed dislocation structures, characteristic of GB and SGB. The angles found here (well below $10^{\circ}$ ) clearly rule out large angle GBs and confirm that these are SGBs (or low angle GBs). The most diffuse variation in a given domain and the misorientation absolute value increases toward the top of the ingot indicating that spread dislocations exist within the subgrains, between the SGBs.
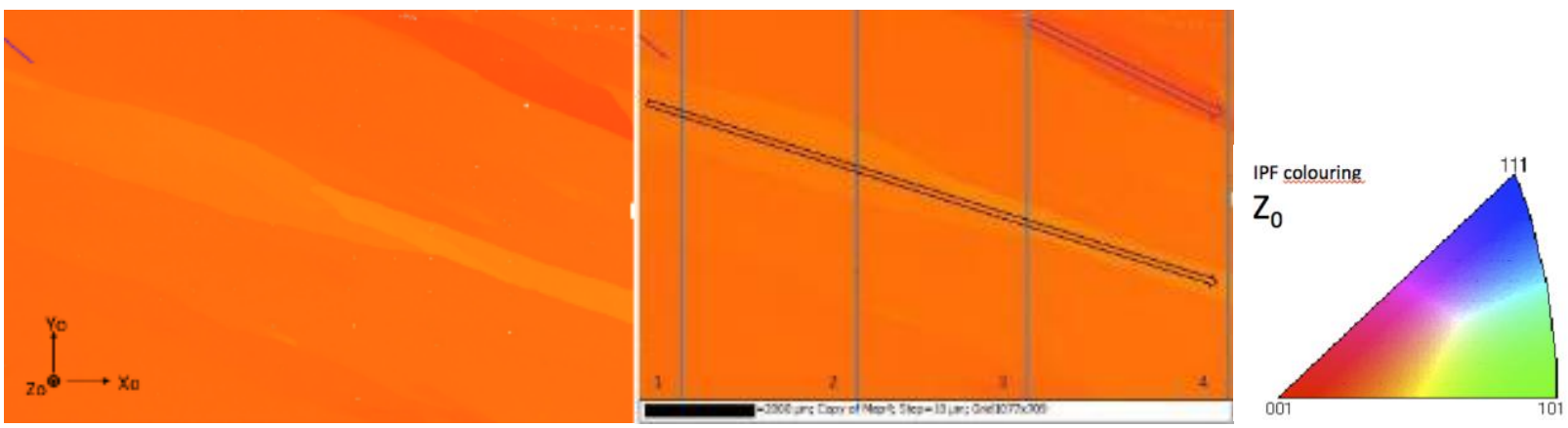
a)

b)

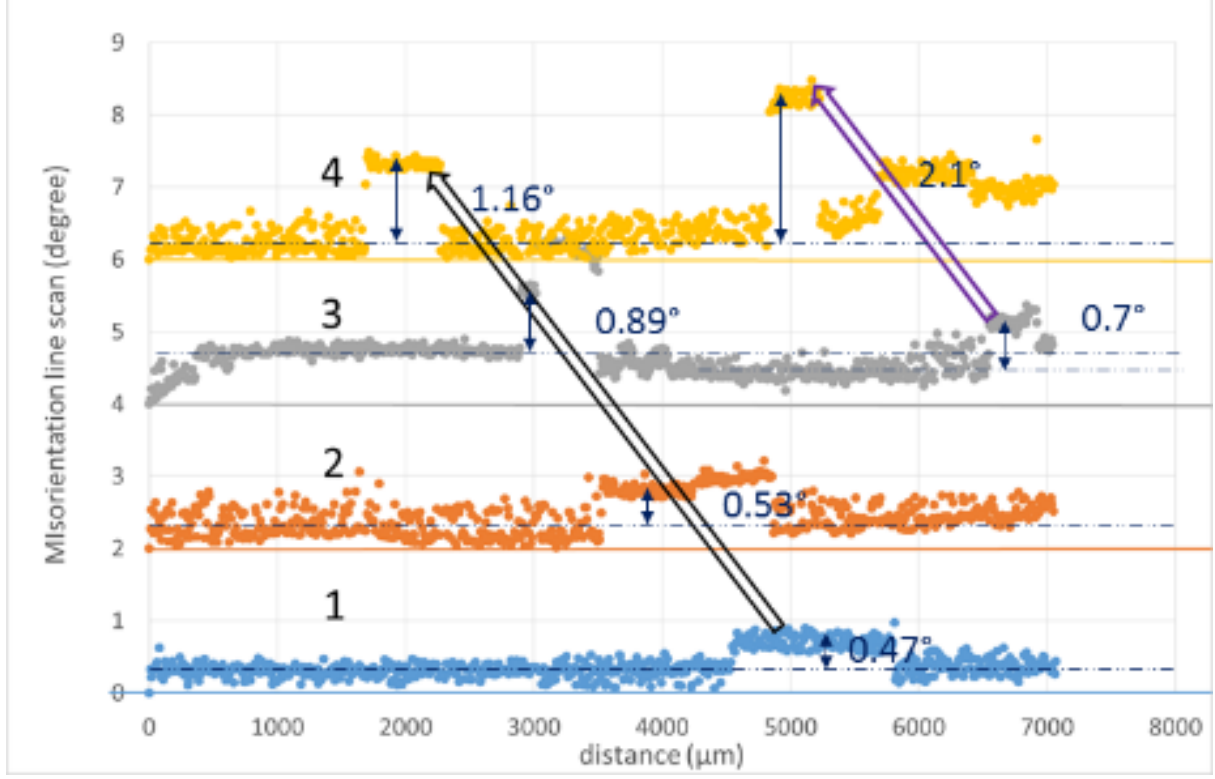

d)

Fig. 5. EBSD map of the sample taken at the top corner of brick B at a height of $180 \mathrm{~cm}$ (B_T22_Z42). a) Crystallographic orientation maps (Inverse Pole Figure color) of the solidified sample in the Zo direction (Xo being the [001] axis close to the growth direction), b) directions of scans, across (1-4) and along (purple and black arrows) the sub-grain domains, c) IPF colouring code, d) plot along Y0 of the absolute misorientations relative to first point (bottom of image b) along the four cross lines (1-4) and identification of corresponding subgrain domains identified by 2 arrows in b). In d), each line scan is shifted by $2^{\circ}$ for clarity.

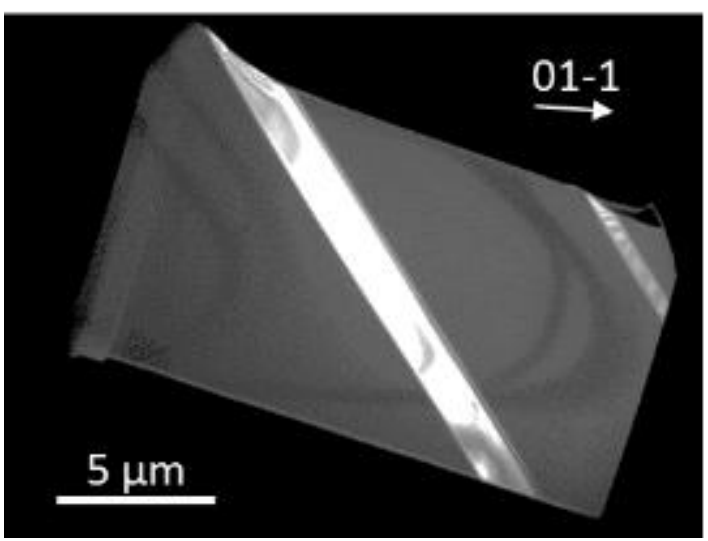

a)

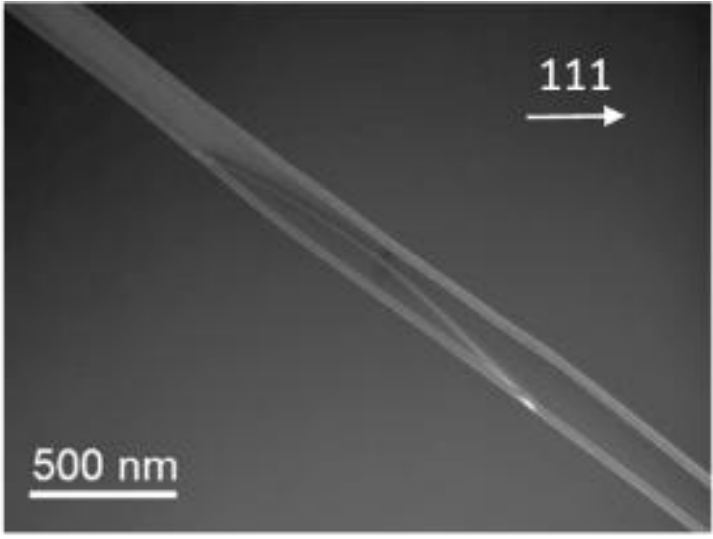

b)

Fig. 6. TEM observation of twinned domains. a) Double twin domain observed in a FIB TEM foil, b) twin domain containing a bowing perfect dislocation in a tensile foil. Arrows indicate the dark field diffraction vector direction.

The observations of twin domains performed so far do not show a strong interaction between eventually mobile dislocations and these twins. Fig. 6 shows that twin domains are often narrow, typically $100 \mathrm{~nm}$ to $2 \mu \mathrm{m}$ wide (Fig. 
6a). They do not contain many dislocations, no pile-ups were observed in their vicinity. Fig. $6 \mathrm{~b}$ is a rare example showing a perfect dislocation (bowed white line) blocked in a twin. We also observed a few individual partial dislocations in these twins, and it is unclear whereas these are the result of an interaction of perfect dislocations with the twin, or growth partial dislocations accommodating stresses during the twin nucleation.

One in-situ TEM straining experiment was performed at decreasing temperatures once dislocations were generated at $1050^{\circ} \mathrm{C}$. The first emitted dislocations originated from a few dislocations present in the foil in a sectioned (by the foil preparation) SGB. At the highest temperature of the experiment $\left(900^{\circ} \mathrm{C}\right.$ and beyond), dislocations behave similarly to regular a/2[110] dislocations gliding in $\{111\}$ planes of a fcc metal. They do not have any specific orientation and glide without experiencing a significant Peierls stress. This is illustrated in Fig. 7a where a dislocation pile-up is seen to progress from the top left corner to the lower right of the picture in the (-1-11) plane (P1) before cross-slipping in the plane (P2) (111). This cross-slip is very likely due to the presence of large precipitates, visible as black regions in Fig. 7a, that are in the path of the pile-up and force most of the dislocations away from this path. Although not characterized in the present thin foil, their size and morphology resemble those of $\mathrm{SiC}$ precipitates that were fully characterized in other TEM foils extracted from the same ingot, so we may infer that these black regions are $\mathrm{SiC}$ precipitates. Because these precipitates are harder than $\mathrm{Si}$, they are able to deviate incoming dislocations. Such a cross-slip event directly gives the Burgers vector of these dislocations, which is the only one contained both in the P1 and P2 planes. The stereographic projection of the crystal during this experiment (Fig. 7b) indicates that $\mathrm{b}=\mathrm{a} / 2$ [101], which corresponds to the intersection of both glide planes. At that point, the foil contains enough dislocations and the temperature can be lowered to 800,720 and $690^{\circ} \mathrm{C}$. The mobile dislocations start adopting a "V" shape, typical of an increasing Peierls stress. Unfortunately, the contamination of the foil during the test also alters the quality of the image. Sketches of the tracked dislocations are represented in Fig. 7f, i. Fig. 7d, e and Fig. 7f, g are snapshots taken respectively at $800^{\circ} \mathrm{C}$ and $720^{\circ} \mathrm{C}$. The corresponding movies can be found in the supplementary material as Movie S1 $\left(800^{\circ} \mathrm{C}\right)$ and Movie S2 $\left(720^{\circ} \mathrm{C}\right)$. These pictures and movies allow us to measure both the velocity of the dislocations and the resolved shear stress acting on them. The stress evaluation is performed by measuring the curvature between the two straight segments (on isolated dislocations). We have used the simplified equation:

$\tau=\mu \mathrm{b} / \mathrm{R}$

where $\tau$ is the local resolved shear stress acting on the mobile dislocation, $\mathrm{b}$ its Burgers vector, $\mathrm{R}$ the radius of curvature (in its glide plane after correction from the projection angle at the given in situ TEM conditions) and $\mu$ the shear modulus of Si (corrected with T). The applied stress $\sigma$ is calculated from $\tau$ knowing the Schmid factor $\mathrm{S}$ for the active slip system:

$\tau=\sigma S$
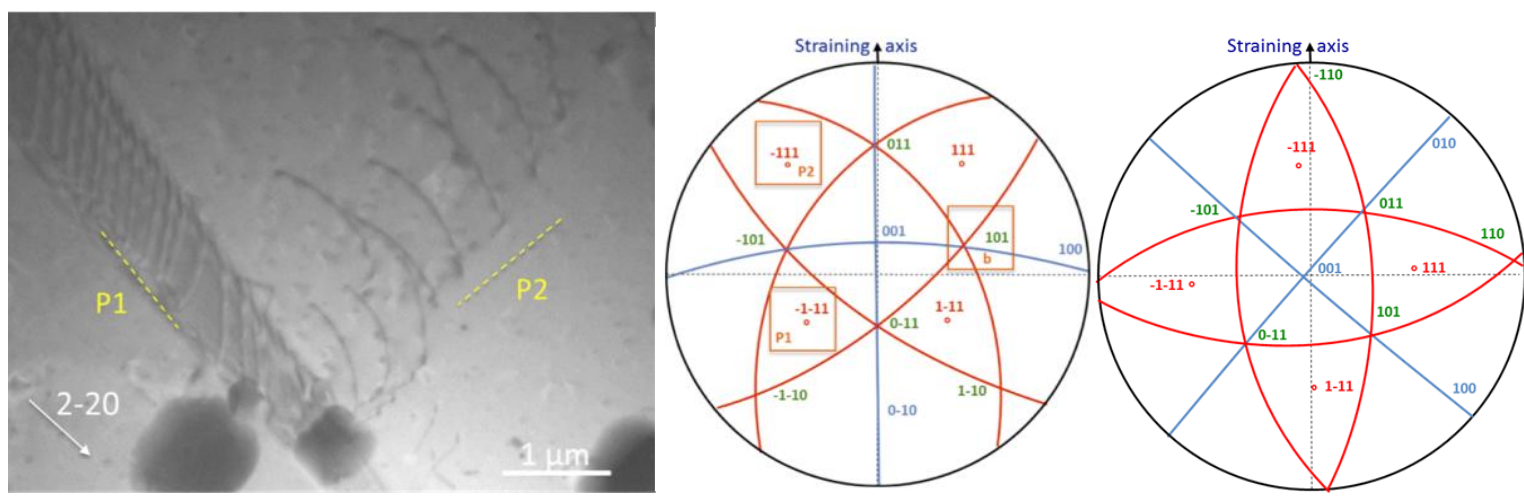
a)

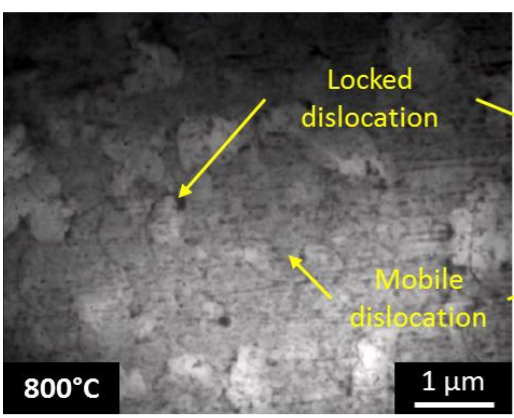

d)

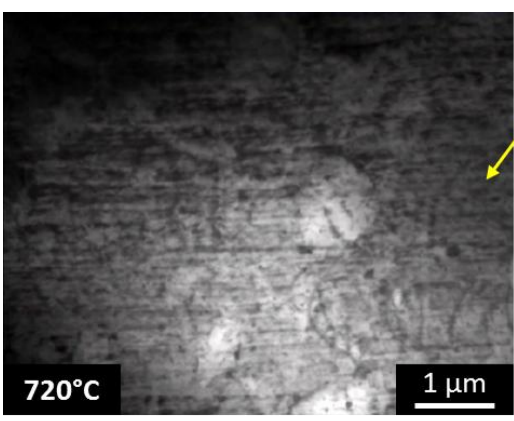

g) b)

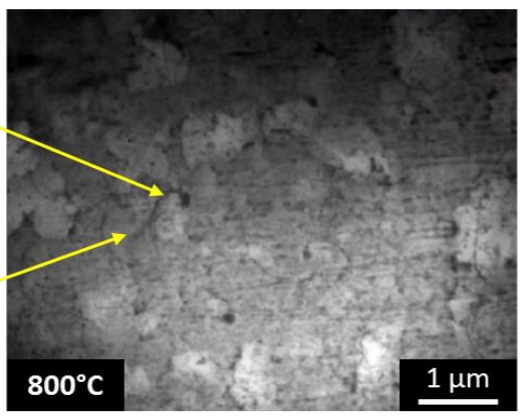

e)

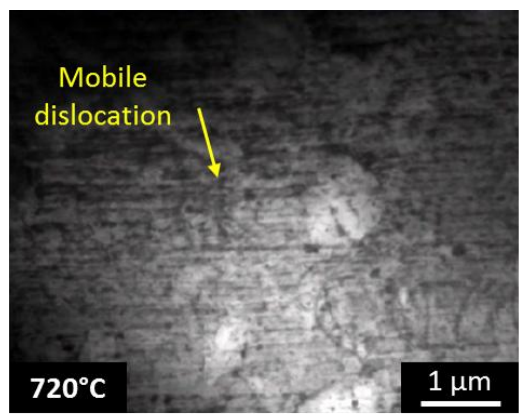

h) c)

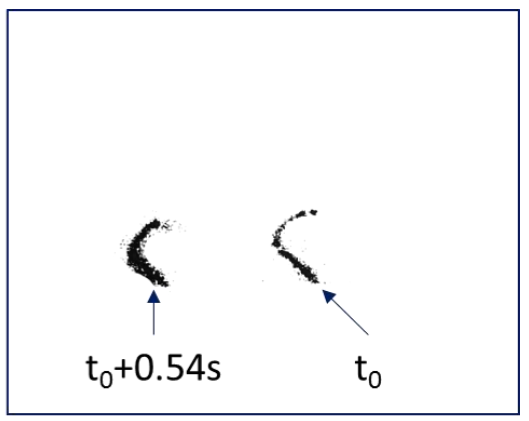

f)

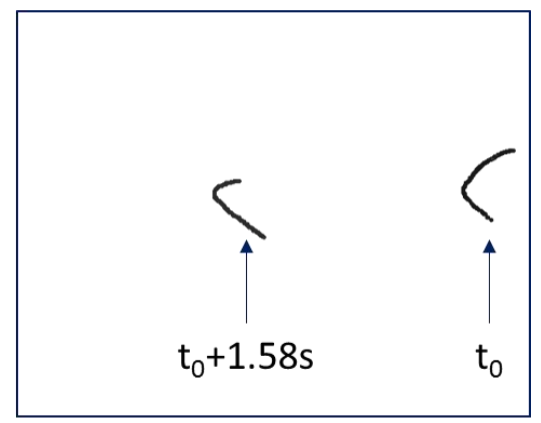

i)

Fig. 7. In-situ TEM observations. a) $\mathrm{T}=900{ }^{\circ} \mathrm{C}$. Screen capture from a video sequence showing a/2[101] dislocations initially gliding on a $\{-1-$ $11\}$ plane $(\mathrm{P} 1)$ and cross slipping on a $\{-111\}$ plane $(\mathrm{P} 2)$; b) corresponding stereographic projection. c) Stereographic projection of video screen captures d,e, g,h; d,e) Video screen captures of a moving dislocation taken $0.54 \mathrm{~s}$ apart showing a dislocation moving from position 0 to 1 at 800 ${ }^{\circ} \mathrm{C}$; g,h) video screen captures showing a dislocation moving from position 0 to 1 in $1.58 \mathrm{~s}$ at $720{ }^{\circ} \mathrm{C}$. Sketch of the moving dislocations in $\mathrm{f}$ ) and i). Images $\mathrm{d}, \mathrm{e}, \mathrm{g} \& \mathrm{~h}$ are extracted from movies $\mathrm{S} 1$ and $\mathrm{S} 2$ that can be found in the supplementary material.

Table 1 regroups our findings related to mobility measurements performed at four temperatures investigated. In this symmetrical configuration (straining axis along [-110], Fig. 7c), the Schmid factors for the 4 slip systems implicated on both $\{-111\}$ and $\{1-11\}$ glide planes are equivalent and equal to 0.42. The dislocations having a [110] Burgers vector direction are unlikely to be active since their Schmid factor is close to 0 on both planes.

\begin{tabular}{|lc|c|c|c|c|}
\hline Temperature & ${ }^{\circ} \mathrm{C}$ & 690 & 720 & 800 & 980 \\
\hline Resolved shear stress & $\mathrm{MPa}$ & 47 & 27 & 26 & 23 \\
\hline Shear modulus & $\mathrm{GPa}$ & 63.2 & 63 & 62.5 & 61.3 \\
\hline \multirow{2}{*}{ Stress } & $\mathrm{MPa}$ & 112 & 64 & 62 & 55 \\
\hline Velocity & & & & & \\
\hline
\end{tabular}

Tab. 1. Dislocation mobility as a function of stress and temperature for the mobile dislocations observed during in-situ TEM experiments. The shear modulus variation with temperature is taken from George [27] after correction of the notorious factor 10 error on the elastic constants in this publication. 


\section{Discussion}

\subsection{Grain boundary structure and misorientations}

When comparing the distances between dislocations of family d1 observed in Fig. 4 and the separation distance between the smallest etch pits in Fig. 3, it can be seen that both correspond exactly. This is why we labelled them alike. There is a bit more discrepancy in the separation distances between the largest etch-pits of Fig. 3, and the distances between dislocations $\mathrm{d} 2$ in Fig. $4(500 \mathrm{~nm}-1 \mu \mathrm{m})$, but this is expected since these dislocations do not have the same regular spacing as $\mathrm{d} 1$. In this study, we haven't been able to further link etch-pits and dislocation type for the third dislocation family identified in TEM (d3), but such an investigation would be interesting as characterizing dislocations from etch pits size and shape would save a lot of time compared to a full TEM analysis [28-30]. It is interesting to note that Oriwol et al [31] concluded that 30-40 nm was the interspacing distance between the individual SGB dislocations that they observed by TEM and that this distance was not resolvable by etch-pits. Here, we have clearly established that the densest SGB dislocations are separated by $90 \mathrm{~nm}$ and that this distance can be also resolved by etch pits observation inside the SEM.

The shape of $\mathrm{d} 2$ dislocations lines that follow the growth direction [001] and the trace of the $\{-1-11\}$ glide plane suggests that these dislocations are either the result of an elastic interaction between gliding a/2[101] dislocations and those from the SGB, or an attractive reaction between gliding a/2[0-1-1] dislocations and the a/2[1-10] dislocations of the $\mathrm{d} 1$ family according to the scheme:

$$
\mathrm{a} / 2[1-10]_{\mathrm{d} 1}-\mathrm{a} / 2[0-1-1]=\mathrm{a} / 2[10-1]_{\mathrm{d} 2}
$$

In both cases, dislocation glide on the $\{-1-11\}$ plane is required. Since the segments remaining along $\{-1-11\}$ are of d2 type, the first hypothesis seems more likely.

Equally, dislocations from the $\mathrm{d} 3$ family $(\mathrm{a} / 2[0-11])$ are all aligned along the $\{111\}$ glide plane and entangled with the two other dislocation families. These three dislocation families are not co-planar. The growth dislocations of d1 and most of the segments of $\mathrm{d} 2$ (also aligned along the growth direction) do not pertain to a glide plane. The potential dissociation of these dislocations was not investigated here as HRTEM would be necessary under various angles. However, dislocations $\mathrm{d} 2$ have a line that pertain to the SGB plane, $\{1-10\}$ in our case, and d1 pertains to $b_{\mathrm{d} 1}$ $\mathrm{x}[001]=[1-10] \mathrm{x}[001]=[-1-10]$, which make them very similar to the Lomer perfect dislocations observed by Bauer et al in multicrystalline solar cells [22]. However, these types of dislocation can also be Lomer-Cotrell locks generated by two intersecting glide planes, in our case $\{-1-11\}$ and $\{111\}$ planes. $\mathrm{d} 1$ and $\mathrm{d} 2$ type dislocations could also be the result of a reaction between the leading and trailing partials of dissociated dislocations gliding in these planes leading to Lomer-Cotrell locks. However, such recombination seems unlikely on a large scale [32].

Since Lomer dislocations have clearly been identified as recombination sites [22], further investigations are needed to see if $\mathrm{d} 1$ or $\mathrm{d} 2$ are to be incriminated for electrical activity. However, as $\mathrm{d} 1$ are clearly sessile dislocations, their propagation along the growth axis indicates that they are maintained by epitaxy growth at the liquid solid interface.

The misorientation of a given SGB is directly function of its denser population of dislocations, that is d1. Knowing the separation distance between the dislocations and their Burgers vector, a simple calculation can lead to an accurate estimation of the SGB misorientation $\theta$ (for small angles):

$$
\sin \theta \approx \theta=\mathrm{b} / \mathrm{d}
$$

Here, $d=91 \mathrm{~nm}$ and $\mathrm{b}=0.384 \mathrm{~nm}$, so $\theta=4.22 \times 10^{-2} \operatorname{Rad}=0.24^{\circ}$.

This very low value is exactly in the range of the SGB misorientations reported elsewhere by TEM [31] or X-rays [21]. Here, he measurement error is only due to the accuracy on the distance between the dislocations (about 2-3nm) 
and can be estimated to less than $0.01^{\circ}$.

The misorientation value is much smaller than the values obtained using EBSD maps (Fig. 5), for several reasons. First, the scales are different. Indeed, the EBSD measurements provide average misorientations over large distances while measurements made on a TEM foil correspond to a small sample of a given SGB. More importantly, the TEM samples have been taken in a lower region of the ingot (height $=90-110 \mathrm{~mm}$ ) where SGB are still building up and do not present high misorientations. They are electrically undetectable because of these very small misorientations that also correspond to small dislocation density. In comparison, the SGBs of Fig. 5 have been observed in upper parts of the ingots (height =180), after having been identified by PL. The fact that they are electrically active indicates that they contain a high density of dislocations, and are therefore, more disoriented. The discrepancy is then expected between SGBs located in the upper part of the ingot, containing large density of dislocations and carrying large misorientations on one hand, and SGBs located at the early stage of growth (ingot bottom), slightly misoriented because their low dislocation density, and therefore electrically harmless on the other hand. Those EBSD maps indicate however a sharp transition between the various angular domains, consistently with the SEM and TEM observations of thin and perfectly ordered SGBs, without clouds of dislocations or cells in their vicinity. The accuracy of the EBSD measurement does not go below $0.1^{\circ}$, which is of the same order of magnitude of misorientation for the individual SGB investigated in Fig. $4\left(0.24^{\circ}\right)$. Both techniques are then complementary at different scales. The evolution of the misorientation along a given domain (or SGB) observed in Fig. 5 could also be explained by the non-negligible proportion of "extrinsic" dislocation families (such as d2 and $\mathrm{d} 3$ in Fig. 4). The fact that the Burgers vector of the main constituent of these SGBs (growth dislocations d1) is purely edge explains the rotation around the growth axis [001]. Variations of the angle amplitude along the growth height can be explained by density changes in d1. Deviations of the tilt direction may be explained by the accumulation of secondary (and perhaps tertiary) dislocations such as $\mathrm{d} 2$ that have a Burgers vector at $45^{\circ}$ from the growth axis.

\subsection{Twins}

As stated earlier, the observed twins do not contain many dislocations, and do not seem to act as strong obstacles to moving dislocations as we haven't observed many pile-ups in their vicinity. One reason for this could be that the dislocations are moving in planes parallel to twins boundaries and therefore do not interact with them. But this hypothesis does not hold when the density of twins is high as in our samples (twins few $\mathrm{mm}$ away in dense parts). In another work [33], we have shown that microtwins follow a macroscopic scheme that move them away from the center of the ingot, accordingly to the convex interface. The fact that we observe very few individual partial dislocations in these twins is also an indication that they do not grow by successive motion of Shockley partials. Their width is also very constant, reinforcing the findings that the twins are grown at the solid/liquid interface as for the case of millimeter scale twinned domains [34] and do not benefit from mechanical twinning that could thicken them once they are created in the solid. Nevertheless, in [34] and in [35] twinning initiates at growth facets from solid/solid/liquid or solid/liquid/gas triple points. Here, microtwins initiate in the interior of the $\approx 15 \times 15 \mathrm{~cm}^{2}$ [100] grain so that no obvious facet source such as a large angle GB is identified. 


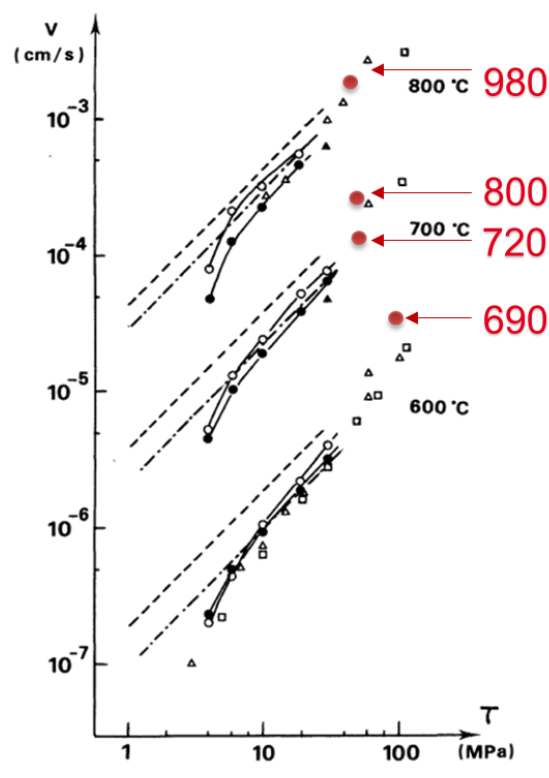

Fig. 8. Comparison of the mobilities found using in-situ TEM in this study (Red dots, temperatures in ${ }^{\circ} \mathrm{C}$ ) and those published for intrinsic $\mathrm{Si}$ ([18]) using etch pits on macroscopic samples.

\subsection{Dislocation mobilities}

A significant literature exists on the effect of doping concentration on dislocation mobility in $\mathrm{Si}$, but the various dopants that have been studied (N, B, P, As, Sb) are usually studied individually [15-17] in Cz or FZ Si. Their concentrations have however been modulated up to $10^{20} \mathrm{~cm}^{-3}$, which is higher than the concentration of $\mathrm{N}, \mathrm{O}$ and $\mathrm{C}$ present in our samples.

Despite the limited data obtained and reported in figure 8, it seems that we observe velocities that would require higher stress or lower temperature than in bulk CZ Si [18]. In the Peierls regime, the mobility is directly dependent on the double-kink mechanism and should not be affected severely by point defect interaction [17], [36], [37]. We haven't observed strong pinning points on mobile dislocations and the large obstacles that could be seen in Fig. 7 locally slow down the dislocation pile-up, but do not affect the local mobility of dislocations away from them. The way the experiments have been performed (strain increments applied to the sample followed by observations made during stress relaxation) could also influence the results, at least in the high temperature regime. At lower temperature (partially explored here), the velocity is much less dependent on the stress [18].

If the double kink mechanism were active in the temperature range investigated, the hardening effect could be attributed to the shorter dislocations segments due to the thin foil configuration: nucleation sites for this mechanism are physically reduced by the length of the truncated dislocation line [38]. In any case, such experiments need to be repeated at different temperatures and by varying the stress range even more. We may also try to perform relaxation tests until the velocity fades off completely to see if a critical stress different from that of pure or doped Si can be evaluated.

The idea of obtaining meaningful velocities values is to be able to establish an order of magnitude of dislocations mean free paths at a given temperature and stress in the ingot during its cooling. A crude calculation here (if we assume, following Fig. 8, that we have the equivalent mobilities of $\mathrm{Cz}$ [18] shifted $100^{\circ} \mathrm{C}$ towards the upper temperatures) would for example give a mobility of $5 \times 10^{-5} \mathrm{~cm} \cdot \mathrm{s}^{-1}$ at $4 \mathrm{MPa}$ at $900^{\circ} \mathrm{C}$. This represents a mean free 
path of $1.8 \mathrm{~mm}$ if the ingot is hold in this state during one hour. Expanding this approximate calculation to the same holding time and stress level to $800^{\circ} \mathrm{C}$ and $1000^{\circ} \mathrm{C}$ would lead to values ten times lower or higher, respectively. That is $18 \mathrm{~mm}$ at $1000^{\circ} \mathrm{C}$ and $180 \mu \mathrm{m}$ at $800^{\circ} \mathrm{C}$. We can conclude that the hourly mean free path of dislocations at a stress of $4 \mathrm{MPa}$ is larger than $1 \mathrm{~mm}$ above $900^{\circ} \mathrm{C}$.

\section{Conclusion}

SEM and TEM observations of dislocations arrangements have been performed in a mono-like Si ingot grown in the [001] direction, with a convex interface.

We have shown that sub-grain boundaries (SGBs) are constituted by a set of dense, perfectly organized immobile dislocations, aligned along the growing direction and having an edge character. These dislocations are typically threading dislocations that are maintained along the growth direction by epitaxial growth at the solid/liquid interface. In our study, the distance between these dislocations, $90 \mathrm{~nm}$, was resolvable both by TEM and by etch pits in high resolution SEM. They lead to a misorientation of about $0.2^{\circ}$, associated with a rotation around the [001] growth axis. These SGBs are probably also responsible for larger misorientations observed using EBSD mapping at higher levels in the ingots. These maps show overall larger misorientations, with sharp transitions and misorientation directions around [001] that are perfectly explained by the organized SGBs characterized by TEM. The amplitude of the misorientation can be explained by the density of their main dislocation constituent, lower at the base and higher when going to the top of the ingot.

These SGBs are decorated by secondary and tertiary dislocation families that are probably generated in the solid state, as indicated by their line directions, that correspond to (111)-type glide plane. One set of these secondary dislocations is incorporated within the SGB by a mechanism that remains to be identified. Their Burgers vectors, at $45^{\circ}$ of the growth direction may explain that misorientations deviates from perfect rotation around the growth axis along the ingots height.

Our observations also show that SGBs are strong obstacles for mobile dislocations, which does not seems to be the case for twins, as those contain very few extrinsic dislocations. Twins were observed to have a fairly constant width along the growth direction, which excluded the possibility of mechanical twinning or absorption of mobile dislocations at a significant level.

Finally, a/2<110>dislocations velocities have been estimated during in-situ TEM straining experiments. They are slightly smaller than those measured in bulk $\mathrm{Cz} \mathrm{Si}$. This could be a thin foil rather than a solid solution effect: no local pinning points that could result from atomic-size defects have been observed. Nonetheless, our experiments show that dislocations are highly mobile above $900^{\circ} \mathrm{C}$ where they might travel several $\mathrm{mm}$ per hour at low stress levels. When these travel distances become much larger than the characteristic microstructural features (twin, cell or SGB) spacing, interactions between dislocations and these potential obstacles cannot be avoided. 


\section{Acknowledgements}

This project has received support from the State Program "Investment for the Future" bearing the reference (ANR -10- IEED -0003)"

\section{References}

[1] F. Jay, D. Muñoz, T. Desrues, E. Pihan, V. Amaral de Oliveira, N. Enjalbert, A. Jouini., "Advanced process for n-type mono-like silicon a-Si:H/c-Si heterojunction solar cells with 21.5\% efficiency," Sol. Energy Mater. Sol. Cells, vol. 130, pp. 690-695, Nov. 2014.

[2] R. Cabal , N. Enjalbert, E. Pihan, N. Plassat, G. Fortin, L. Bounaas, S. Dubois, "CRYSTALMAX Silicon for High Efficiency / Low-Cost Solar Cells," presented at the 32nd European Photovoltaic Solar Energy Conference and Exhibition, 2016, pp. 285 - 288.

[3] N. Stoddard, B. Gründig-Wendrock, A. Krause, D. Oriwol, M. Bertoni, T.U. Naerland, I. Witting, L. Sylla, “On the potential and limits of large area seeding for photovoltaic silicon,” J. Cryst. Growth, no. 452, pp. 272-275, 2016.

[4] I. Guerrero,V. Parra, T. Carballo,A. Black,M. Miranda,D. Cancillo, B. Moralejo, J. Jiménez, J-F. Lelièvre, C. del Cañizo, "About the origin of low wafer performance and crystal defect generation on seed-cast growth of industrial mono-like silicon ingots: Seed-cast growth of industrial mono-like silicon ingots," Prog. Photovolt. Res. Appl., vol. 22, no. 8, pp. 923-932, Aug. 2014.

[5] Y. Zhang, Z. Li, Q. Meng, Z. Hu, and L. Liu, "Distribution and propagation of dislocation defects in quasi-single crystalline silicon ingots cast by the directional solidification method," Sol. Energy Mater. Sol. Cells, vol. 132, pp. 1-5, Jan. 2015.

[6] K. Jiptner, Y. Miyamura, H. Harada, B. Gao, K. Kakimoto, and T. Sekiguchi, "Dislocation behavior in seed-cast grown Si ingots based on crystallographic orientation: Dislocation behavior in seed-cast grown Si ingots," Prog. Photovolt. Res. Appl., vol. 24, no. 12, pp. 1513-1522, Dec. 2016.

[7] A. Krause, L. Sylla, and D. Oriwol, "Plastic Deformation as an Origin of Dislocations in Cast Mono," Energy Procedia, vol. 92, pp. 833-838, Aug. 2016.

[8] V. Amaral de Oliveira, M. Tsoutsouva, T. Lafford, E. Pihan, F. Barou, C. Cayron, D. Camel., "Sub-Grain Boundaries Sources And Effects in Large Mono-Like Silicon Ingots For PV," presented at the 29th European Photovoltaic Solar Energy Conference and Exhibition, 2014, pp. 793 - 797. [9] Trempa, M., Beier, M., Reimann, C., Roßhirth, K., Friedrich, J., Löbel, C., Sylla, L., Richter, T.,, "Dislocation formation in seed crystals induced by feedstock indentation during growth of quasimono crystalline silicon ingots," J. Cryst. Growth, vol. 454, pp. 6-14, Nov. 2016.

[10] V. Amaral De Oliveira, "Influence of processing parameters on the generation and propagation of electrically active crystalline defects in monolike silicon," Thèse de doctorat, Université de Grenoble, France, 2016.

[11] P. Rudolph, "Dislocation patterning and bunching in crystals and epitaxial layers - a review: Review paper in honor of that 50. Anniversary of CRT," Cryst. Res. Technol., vol. 52, no. 1, p. 1600171, Jan. 2017.

[12] H. Alexander and P. Haasen, "Dislocations and Plastic Flow in the Diamond Structure," in Solid State Physics, vol. 22, Elsevier, 1969, pp. 27-158.

[13] K. Sumino, "A model for the dynamical state of dislocations in crystals during deformation," Mater. Sci. Eng., vol. 13, no. 3, pp. 269-275, Mar. 1974.

[14] M. Suezawa, K. Sumino, and I. Yonenaga, "Dislocation dynamics in the plastic deformation of silicon crystals. II. Theoretical analysis of experimental results," Phys. Status Solidi A, vol. 51, no. 1, pp. 217-226, Jan. 1979. 
[15] Yonenaga, "Activities of dislocations in heavily impurity-doped Si," J. Phys. Condens. Matter, vol. 12, no. 49, p. 10065, 2000.

[16] I. Yonenaga, "Dislocation behavior in heavily impurity doped Si," Scr. Mater., vol. 45, no. 11, pp. 1267-1272, Nov. 2001.

[17] I. Yonenaga, "Dislocation-impurity interaction in Si," Mater. Sci. Eng. B, vol. 124-125, pp. 293-296, Dec. 2005.

[18] A. George and J. Rabier, "Dislocations and plasticity in semiconductors. I - Dislocation structures and dynamics," Rev. Phys. Appliquée, vol. 22, no. 9, pp. 941-966, 1987.

[19] M. M. Kivambe, G. Stokkan, T. Ervik, B. Ryningen, and O. Lohne, "The microstructure of dislocation clusters in industrial directionally solidified multicrystalline silicon," J. Appl. Phys., vol. 110, no. 6, p. 063524, Sep. 2011.

[20] M. M. Kivambe, T. Ervik, B. Ryningen, and G. Stokkan, "On the role of stacking faults on dislocation generation and dislocation cluster formation in multicrystalline silicon," J. Appl. Phys., vol. 112, no. 10, p. 103528, 2012.

[21] D. Oriwol, E.-R. Carl, A.N. Danilewsky, L. Sylla, W. Seifert, M. Kittler, H.S. Leipner,, "Small-angle subgrain boundaries emanating from dislocation pile-ups in multicrystalline silicon studied with synchrotron white-beam X-ray topography," Acta Mater., vol. 61, no. 18, pp. 6903-6910, Oct. 2013.

[22] J. Bauer, A. Hahnel, P. Werner, N. Zakharov, H. Blumtritt, A. Zuschlag, O. Breitenstein, "Recombination at Lomer Dislocations in Multicrystalline Silicon for Solar Cells," IEEE J. Photovolt., vol. 6, no. 1, pp. 100-110, 2016.

[23] J. Edington, Interpretation of Transmission Electron Micrographs, vol. 3 of Monographs in Practical Electron Microscopy in Materials Science, Chap. III. Philips Technical Library, 1976.

[24] M. Legros, O. Ferry, F. Houdellier, A. Jacques, and A. George, "Fatigue of single crystalline silicon: Mechanical behaviour and TEM observations," Mater. Sci. Eng. A, vol. 483-484, pp. 353-364, Jun. 2008.

[25] A. George, A. Jacques, and M. Legros, "Low-cycle fatigue in silicon: comparison with fcc metals," Fatigue Fract. Eng. Mater. Struct., vol. 30, no. 1, pp. 41-56, Jan. 2007.

[26] M. W. Jenkins, “A New Preferential Etch for Defects in Silicon Crystals," J. Electrochem. Soc., vol. 124, no. 5, p. 757, 1977.

[27] R. Hull, Properties of Crystalline Silicon. INSPEC, the Institution of Electrical Engineers, 1999.

[28] J. J. Gilman, W. G. Johnston, and G. W. Sears, "Dislocation Etch Pit Formation in Lithium Fluoride," J. Appl. Phys., vol. 29, no. 5, pp. 747-754, May 1958.

[29] T. Hino, S. Tomiya, T. Miyajima, K. Yanashima, S. Hashimoto, and M. Ikeda, "Characterization of threading dislocations in GaN epitaxial layers," Appl. Phys. Lett., vol. 76, no. 23, pp. 3421-3423, Jun. 2000.

[30] A. Tallaire et al., "Identification of Dislocations in Synthetic Chemically Vapor Deposited Diamond Single Crystals," Cryst. Growth Des., vol. 16, no. 5, pp. 2741-2746, May 2016.

[31] D. Oriwol, M. Trempa, L. Sylla, and H. S. Leipner, "Investigation of dislocation cluster evolution during directional solidification of multicrystalline silicon," J. Cryst. Growth, vol. 463, pp. 1-9, Apr. 2017.

[32] A. Bourret, J. Desseaux, and A. Renault, "Core structure of the Lomer dislocation in germanium and silicon," Philos. Mag. A, vol. 45, no. 1, pp. 1-20, Jan. 1982.

[33] A. Lantreibecq, E. Pihan, N. Plassat, and M. Legros, "Spatial distribution of structural defects in Cz-seeded directionally solidified silicon ingots: An etch pit study," J. Cryst. Growth Be Submitt.

[34] T. Riberi-Béridot, N. Mangelinck-Noël, A. Tandjaoui, G. Reinhart, B. Billia, T. Lafford, J. Baruchel, L Barrallier, "On the impact of twinning on the formation of the grain structure of multi-crystalline silicon for photovoltaic applications during directional solidification," J. Cryst. Growth, vol. 418, pp. 38-44, May 2015.

[35] M.G. Tsoutsouva , T. Riberi-Béridot , G. Regula , G. Reinhart , J. Baruchel , F. Guittonneau , L. Barrallier , and N. Mangelinck-Nöel, "In situ investigation of the structural defect generation and evolution during the directional solidification of $\langle 110\rangle$ seeded growth Si," Acta Mater., vol. 115, pp. 210-223, Aug. 2016.

[36] D. Caillard, "A TEM in situ study of alloying effects in iron. I-Solid solution softening caused by low concentrations of Ni, Si and Cr," Acta Mater., vol. 61, no. 8, pp. 2793-2807, May 2013.

[37] D. Caillard, "A TEM in situ study of alloying effects in iron. II-Solid solution hardening caused by high 
concentrations of Si and Cr," Acta Mater., vol. 61, no. 8, pp. 2808-2827, May 2013.

[38] F. Louchet, J. Pelissier, D. Caillard, J. P. Peyrade, C. Levade, and G. Vanderschaeve, "In situ TEM study of dislocation mobility in semiconducting materials," Microsc. Microanal. Microstruct., vol. 4, no. 2-3, pp. 199210, 1993.

\section{Supplementary Material}

\section{Annex 1 - Indexation of dislocation families and stereographic projections.}

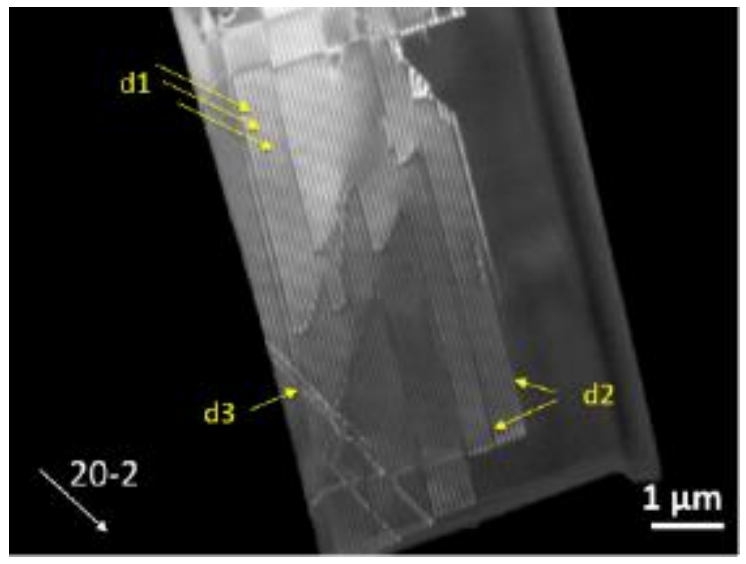

a)

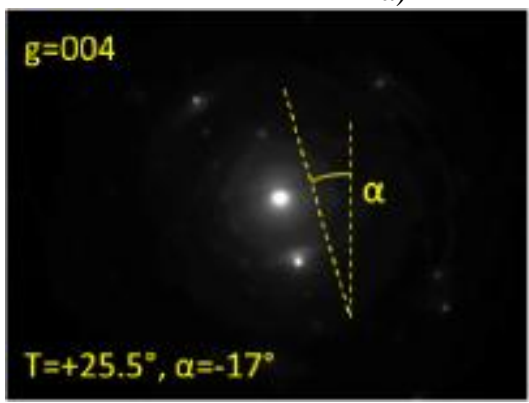

c)

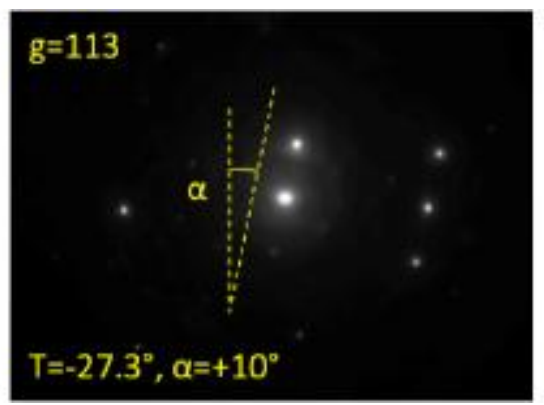

d)

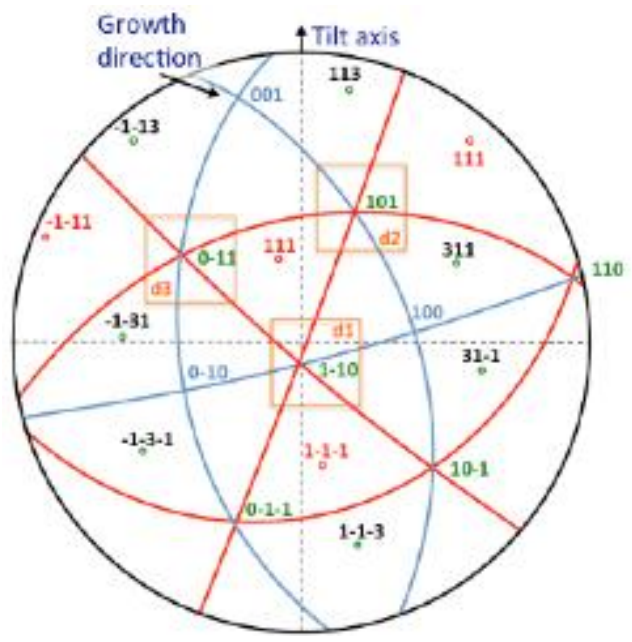

b)

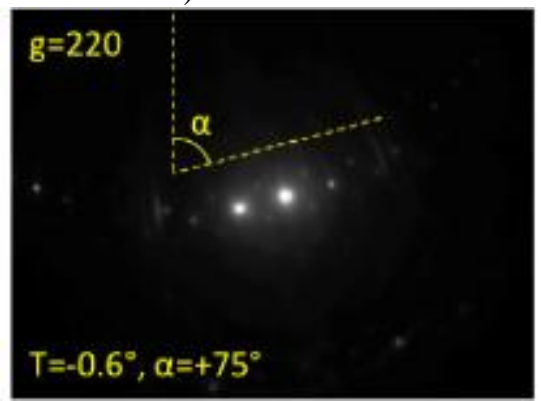

e)

Fig. S1. Analysis of SGB dislocations (following Fig.4). a) Dark field micrograph using the 20-2 reflection of the SGB showing the d2 family out of contrast, while d1 and $\mathrm{d} 3$ are clearly visible, b) stereographic projection corresponding to the orientation of the foil in a) and constructed from 7 two-beam conditions from which c) $g=004$, d) $g=113$ and e) $g=220$ are examples. $\alpha$ represent the angle between the vertical axis on the diffraction pattern that also corresponds to the holder tilting axis (labelled T) that is also the straining axis for the straining holder.

Annex 2 - Dynamic observations of moving dislocations.

Movie S1: In situ TEM. Movements of a/2<110> perfect dislocations on a $\{111\}$ plane at $800{ }^{\circ} \mathrm{C}$ in a sample under stress.

Movie S1: In situ TEM Movements of a/2<110> perfect dislocations on a $\{111\}$ plane at $720{ }^{\circ} \mathrm{C}$ in a sample under 
stress.

The corresponding stereographic projection for these sequences is Fig. 7c. 